\title{
BRODY CURVES OMITTING HYPERPLANES
}

\author{
Alexandre Eremenko \\ Purdue University, Department of Mathematics \\ West Lafayette, IN 47907-2067, U.S.A.; eremenko@math.purdue.edu
}

\begin{abstract}
A Brody curve, a.k.a. normal curve, is a holomorphic map $f$ from the complex line $\mathbf{C}$ to the complex projective space $\mathbf{P}^{n}$ such that the family of its translations $\{z \mapsto f(z+a): a \in \mathbf{C}\}$ is normal. We prove that Brody curves omitting $n$ hyperplanes in general position have growth order at most one, normal type. This generalizes a result of Clunie and Hayman who proved it for $n=1$.
\end{abstract}

\section{Introduction}

We consider holomorphic curves $f: \mathbf{C} \rightarrow \mathbf{P}^{n}$. The spherical derivative $\left\|f^{\prime}\right\|$ measures the length distortion from the Euclidean metric in $\mathbf{C}$ to the Fubini-Study metric in $\mathbf{P}^{n}$. The explicit expression is

$$
\left\|f^{\prime}\right\|^{2}=\|f\|^{-4} \sum_{i<j}\left|f_{i}^{\prime} f_{j}-f_{i} f_{j}^{\prime}\right|^{2}
$$

where $\left(f_{0}, \ldots, f_{n}\right)$ is a homogeneous representation of $f$ (that is the $f_{j}$ are entire functions which never simultaneously vanish), and

$$
\|f\|^{2}=\sum_{j=0}^{n}\left|f_{j}\right|^{2} .
$$

A holomorphic curve is called a Brody curve if its spherical derivative is bounded. This is equivalent to normality of the family of translations $\{z \mapsto f(z+a): a \in \mathbf{C}\}$.

Brody curves are important for at least two reasons. First one is the rescaling trick known as Zalcman's lemma or Brody's lemma: for every non-constant holomorphic curve $f$ one can find a sequence of affine maps $a_{k}: \mathbf{C} \rightarrow \mathbf{C}$ such that the limit $f \circ a_{k}$ exists and is a non-constant Brody curve. Second reason is Gromov's theory of mean dimension [4] in which a space of Brody curves is one of the main examples.

For the recent work on Brody curves we refer to $[3,9,10,12,13]$. A general reference for holomorphic curves is [6].

We recall that the Nevanlinna characteristic is defined by

$$
T(r, f)=\int_{0}^{r} \frac{d t}{t}\left(\frac{1}{\pi} \int_{|z| \leq t}\left\|f^{\prime}\right\|^{2}(z) d m_{z}\right),
$$

where $d m$ is the area element in $\mathbf{C}$. So Brody curves have order at most two normal type, that is

$$
T(r, f)=O\left(r^{2}\right)
$$

doi:10.5186/aasfm.2010.3534

2000 Mathematics Subject Classification: Primary 32Q99, 30D15.

Key words: Holomorphic curve, spherical derivative.

The author supported by NSF grant DMS-0555279 and by the Humboldt Foundation. 
Clunie and Hayman [2] found that Brody curves $\mathbf{C} \rightarrow \mathbf{P}^{1}$ omitting one point in $\mathbf{P}^{1}$ must have smaller order of growth:

$$
T(r, f)=O(r) .
$$

A different proof of this fact is due to Pommerenke [8]. In this paper we prove that this phenomenon persists in all dimensions.

Theorem. Brody curves $f: \mathbf{C} \rightarrow \mathbf{P}^{n}$ omitting $n$ hyperplanes in general position satisfy (2).

Under the stronger assumption that a Brody curve omits $n+1$ hyperplanes in general position, the same conclusion was obtained by Berteloot and Duval [1] and Tsukamoto [9], with different proofs.

Combined with a result of Tsukamoto [10] our theorem implies

Corollary. Mean dimension in the sense of Gromov of the space of Brody curves in

$$
\mathbf{P}^{n} \backslash\{n \text { hyperplanes in general position }\}
$$

is zero.

The condition that $n$ hyperplanes are omitted is exact: it is easy to show by direct computation that the curve $\left(f_{0}, f_{1}, 1, \ldots, 1\right)$, where $f_{i}$ are appropriately chosen entire functions such that $f_{1} / f_{0}$ is an elliptic function, is a Brody curve, it omits $n-1$ hyperplanes, and $T(r, f) \sim c r^{2}, r \rightarrow \infty$ where $c>0$. This example will be discussed in the end of the paper.

The author thanks Alexandr Rashkovskii and Masaki Tsukamoto for inspiring conversations on the subject.

\section{Preliminaries}

Without loss of generality we assume that the omitted hyperplanes are given in the homogeneous coordinates by the equations $\left\{w_{j}=0\right\}, 1 \leq j \leq n$. We fix a homogeneous representation $\left(f_{0}, \ldots, f_{n}\right)$ of our curve, where $f_{j}$ are entire functions without common zeros, and $f_{n}=1$. We assume without loss of generality that $f_{0}(0) \neq 0$.

Then

$$
u=\log \sqrt{\left|f_{0}\right|^{2}+\ldots+\left|f_{n}\right|^{2}}
$$

is a positive subharmonic function, and Jensen's formula gives

$$
T(r, f)=\frac{1}{2 \pi} \int_{-\pi}^{\pi} u\left(r e^{i \theta}\right) d \theta-u(0)=\int_{0}^{r} \frac{n(t)}{t} d t,
$$

where $n(t)=\mu(\{z:|z| \leq t\})$, and $\mu$ is the Riesz measure of $u$, that is the measure with the density

$$
\frac{1}{2 \pi} \Delta u=\frac{1}{\pi}\left\|f^{\prime}\right\|^{2}
$$

Now positivity of $u$ and (1) imply that all $f_{j}$ are of order at most 2 , normal type.

In particular,

$$
f_{j}=e^{P_{j}}, \quad 1 \leq j \leq n,
$$

where $P_{j}$ are polynomials of degree at most two. 
First we state a lemma which is the core of our arguments. It is a refined version of Lemma 1 in [2]. We denote by $B(a, r)$ the open disc of radius $r$ centered at the point $a$.

Lemma 1. Let $u$ be a non-negative harmonic function in the closure of the disc $B(a, R)$, and assume that $u\left(z_{1}\right)=0$ for some point $z_{1} \in \partial B(a, R)$. Then

$$
\left|\nabla u\left(z_{1}\right)\right| \geq \frac{u(a)}{2 R} .
$$

Proof. The function

$$
b(r)=\min _{|z-a|=r} u(z)
$$

is decreasing and $b(R)=0$. Harnack's inequality gives

$$
b(t) \geq \frac{R-t}{R+t} u(a), \quad 0 \leq t \leq R .
$$

As

$$
b(t)=|b(R)-b(t)| \leq(R-t) \max _{[t, R]}\left|b^{\prime}\right|,
$$

we conclude that for every $t \in(0, R)$ there exists $r \in[t, R]$ such that

$$
\left|b^{\prime}(r)\right| \geq \frac{1}{R-t} \frac{R-t}{R+t} u(a)=\frac{u(a)}{R+t} .
$$

According to Hadamard's three circle theorem, $r b^{\prime}(r)$ is a negative decreasing function, so

$$
\left|R b^{\prime}(R)\right| \geq\left|r b^{\prime}(r)\right| \geq r \frac{u(a)}{R+t} \geq t \frac{u(a)}{R+t},
$$

and the last expression tends to $u(a) / 2$ as $t \rightarrow R$. So we have $\left|b^{\prime}(R)\right| \geq u(a) /(2 R)$. On the other hand, $\left|\nabla u\left(z_{1}\right)\right| \geq\left|\frac{d u}{d n}\left(z_{1}\right)\right| \geq\left|b^{\prime}(R)\right|$, where $d / d n$ is the normal derivative. This completes the proof.

\section{Proof of the theorem}

We may assume without loss of generality that $f_{0}$ has at least one zero. Indeed, we can compose $f$ with an automorphism of $\mathbf{P}^{n}$, for example replace $f_{0}$ by $f_{0}+c f_{1}, c \in \mathbf{C}$ and leave all other $f_{j}$ unchanged. This transformation changes neither the $n$ omitted hyperplanes nor the rate of growth of $T(r, f)$ and multiplies the spherical derivative by a bounded factor.

Put $u_{j}=\log \left|f_{j}\right|$, and

$$
u^{*}=\max _{1 \leq j \leq n} u_{j}
$$

Here and in what follows max denotes the pointwise maximum of subharmonic functions. We are going to prove first that

$$
u_{0}(z) \leq u^{*}(z)+4(n+1)|z| \sup _{\mathbf{C}}\left\|f^{\prime}\right\| .
$$

for $|z|$ sufficiently large. 
Let $a$ be a point such that $u_{0}(a)>u^{*}(a)$. Consider the maximal disc $B(a, R)$ centered at $a$ where the inequality $u_{0}(z)>u^{*}(z)$ still holds. If $z_{0}$ is a zero of $f_{0}$, then $u_{0}\left(z_{0}\right)=-\infty$, and we have

$$
R \leq|a|+\left|z_{0}\right| \leq 2|a|
$$

for $|a|>\left|z_{0}\right|$. There is a point $z_{1} \in \partial B(a, R)$ and an integer $k \in\{1, \ldots, n\}$ such that

$$
u_{0}\left(z_{1}\right)=u^{*}\left(z_{1}\right)=u_{k}\left(z_{1}\right) \geq u_{j}\left(z_{1}\right)
$$

for all $j \in\{1, \ldots, n\}$. Applying Lemma 1 to the positive harmonic function $u_{0}-u_{k}$ in $B(a, R)$ we obtain

$$
\left|\nabla\left(u_{0}-u_{k}\right)\left(z_{1}\right)\right| \geq \frac{u_{0}(a)-u_{k}(a)}{2 R}
$$

or

$$
u_{0}(a) \leq u_{k}(a)+2 R\left|\nabla u_{0}\left(z_{1}\right)-\nabla u_{k}\left(z_{1}\right)\right| .
$$

On the other hand, $\left|f_{0}\left(z_{1}\right)\right|=\left|f_{k}\left(z_{1}\right)\right| \geq\left|f_{j}\left(z_{1}\right)\right|$ for all $j \in\{1, \ldots, n\}$, so

$$
\left\|f^{\prime}\left(z_{1}\right)\right\| \geq \frac{\left|f_{0}^{\prime}\left(z_{1}\right) f_{k}\left(z_{1}\right)-f_{0}\left(z_{1}\right) f_{k}^{\prime}\left(z_{1}\right)\right|}{\left|f_{0}\left(z_{1}\right)\right|^{2}+\ldots+\left|f_{n}\left(z_{1}\right)\right|^{2}} \geq(n+1)^{-1}\left|\frac{f_{0}^{\prime}\left(z_{1}\right)}{f_{0}\left(z_{1}\right)}-\frac{f_{k}^{\prime}\left(z_{1}\right)}{f_{k}\left(z_{1}\right)}\right| .
$$

Combining (8), (9) and (6), and taking into account that $|\nabla \log | f||=\left|f^{\prime} / f\right|$, we obtain (5).

If all polynomials $P_{j}$ are linear then inequality (5) completes the proof. Suppose now that some $P_{j}$ is of degree 2 .

Consider again the subharmonic functions $u_{j}=\log \left|f_{j}\right|, 0 \leq j \leq n$. For each $j \in\{0, \ldots, n\}$, the family

$$
\left\{r^{-2} u_{j}(r z): r>1\right\}
$$

in uniformly bounded from above on compact subsets of the plane, and bounded from below at 0 . By [5, Theorem 4.1.9] these families are normal (from every sequence one can choose a subsequence that converges in $L_{\text {loc }}^{1}$ ). Take a sequence $r_{k}$ such that

$$
\lim _{k \rightarrow \infty} \frac{1}{r_{k}^{2}} \int_{-\pi}^{\pi} u\left(r_{k} e^{i \theta}\right) d \theta>0
$$

where $u$ is defined in (3). Such sequence exists because we assume that at least one of the $P_{j}$ is of degree two.

Then we choose a subsequence (still denoted by $r_{k}$ ) such that

$$
r_{k}^{-2} u_{j}\left(r_{k} z\right) \rightarrow v_{j}, \quad 0 \leq j \leq n,
$$

and $r_{k}^{-2} u\left(r_{k} z\right) \rightarrow v$, where $v_{j}, v$ are some subharmonic functions in $\mathbf{C}$. Then

$$
v=\max \left\{v_{0}, \ldots, v_{n}\right\} \neq 0
$$

is a non-negative subharmonic function. Let $\nu$ be the Riesz measure of $v$. Notice that $\nu \neq 0$ because $v$ is non-negative and $v \neq 0$. We have weak convergence

$$
\nu=\lim _{k \rightarrow \infty} \mu_{r_{k}},
$$

where

$$
\mu_{r_{k}}(E)=r_{k}^{-2} \mu\left(r_{k} E\right)
$$

for every Borel set $E$. Now (4) and the condition that $\left\|f^{\prime}\right\|$ is bounded imply 
Lemma 2. $\nu$ is absolutely continuous with respect to Lebesque's measure in the plane, with bounded density.

Proof. For every disc $B(a, \delta)$ we have

$$
\nu(B(a, \delta)) \leq \liminf _{k \rightarrow \infty} r_{k}^{-2} \mu\left(B\left(r_{k} a, r_{k} \delta\right)\right) \leq \delta^{2} \sup _{\mathbf{C}}\left\|f^{\prime}\right\|^{2} .
$$

Now we invoke our inequality (5). It implies that

$$
v_{0} \leq v^{*}=\max \left(v_{1}, \ldots, v_{n}\right),
$$

so $v=v^{*}$. Thus the measure $\nu$ is supported by finitely many rays. This contradiction with Lemma 2 shows that all polynomials $P_{j}$ are in fact linear. This completes the proof.

\section{Example}

Let $\Gamma_{0}=\{n+i m: n, m \in \mathbf{Z}\}$ be the integer lattice in the plane, and $\Gamma_{1}=$ $\Gamma+(1+i) / 2$. For $j \in\{0,1\}$, let $f_{j}$ be the Weierstrass canonical products of genus 2 with simple zeros on $\Gamma_{j}$. Then the $f_{j}$ are entire functions of completely regular growth in the sense of Levin-Pfluger and their zeros satisfy the $R$-condition in [7, Theorem 5, Ch. 2]. This theorem of Levin implies that

$$
\log \left|f_{j}\left(r e^{i \theta}\right)\right|=(c+o(1)) r^{2},
$$

as $r \rightarrow \infty, r e^{i \theta} \notin C_{0}$ where $C_{0}$ is a union of discs of radius $1 / 4$ centered at the zeros of $f_{j}$. It follows that

$$
\left|f_{0}(z)\right|^{2}+\left|f_{1}(z)\right|^{2} \rightarrow \infty, \quad z \rightarrow \infty
$$

Cauchy's estimate for the derivative and (11) give

$$
\log \left|f_{j}^{\prime}(z)\right| \leq(c+o(1))|z|^{2}, \quad z \rightarrow \infty .
$$

So for the curve $f=\left(f_{0}, f_{1}, 1, \ldots, 1\right)$ we obtain

$$
\begin{aligned}
\left\|f^{\prime}\right\|^{2} & =\frac{\sum_{i \neq j}\left|f_{i}^{\prime} f_{j}-f_{i} f_{j}^{\prime}\right|^{2}}{\|f\|^{4}} \leq \frac{\left(\left|f_{0}^{\prime} f_{1}-f_{0} f_{1}^{\prime}\right|^{2}+n\left(\left|f_{0}^{\prime}\right|^{2}+\left|f_{1}^{\prime}\right|^{2}\right)\right)}{\left(\left|f_{0}\right|^{2}+\left|f_{1}\right|^{2}\right)^{2}} \\
& =\frac{\left|g^{\prime}\right|^{2}}{\left(1+|g|^{2}\right)^{2}}+o(1) .
\end{aligned}
$$

The spherical derivative of $g$ is bounded because $g$ is an elliptic function. Thus $f$ is a Brody curve that omits $n-1$ hyperplanes in general position. Evidently $T(r, f) \sim c_{1} r^{2}$.

\section{References}

[1] Berteloot, F., and J. Duval: Sur l'hyperbolicité de certains complémentaires. - Enseign. Math. (2) 47:3-4, 2001, 253-267.

[2] Clunie, J., and W. Hayman: The spherical derivative of integral and meromorphic functions. - Comment. Math. Helv. 40, 1966, 373-381.

[3] Eremenko, A.: Normal holomorphic curves from parabolic regions to projective spaces. arXiv:0710.1281.

[4] Gromov, M.: Topological invariants of dynamical systems and spaces of harmonic maps. Math. Phys. Anal. Geom. 2:4, 1999, 323-415. 
[5] Hörmander, L.: Analysis of linear partial differential operators I. Distribution theory and Fourier analysis. - Springer, Berlin, 1983.

[6] LANG, S.: Introduction to complex hyperbolic spaces. - Springer-Verlag, New York, 1987.

[7] Levin, B.: Distribution of zeros of entire functions. - AMS, Providence, RI, 1980.

[8] Pommerenke, Ch.: Estimates for normal meromorphic functions. - Ann. Acad. Sci. Fenn. Ser. A I Math. 476, 1970.

[9] Tsukamoto, M.: On holomorphic curves in algebraic torus. - J. Math. Kyoto Univ. 47:4, $2007,881-892$.

[10] Tsukamoto, M.: Moduli space of Brody curves, energy and mean dimension. - Nagoya Math. J. 192, 2008, 27-58.

[11] Tsukamoto, M.: A packing problem for holomorphic curves. - Nagoya Math. J. 194, 2009, $22-68$.

[12] Tsukamoto, M.: Deformation of Brody curves and mean dimension. - Ergodic Theory Dynam. Systems 29:5, 2009, 1641-1657.

[13] Winkelmann, J.: On Brody and entire curves. - Bull. Soc. Math. France 135:1, 2007, 25-46.

Received 8 October 2009 